\title{
Systemic calciphylaxis presenting as a painful, proximal myopathy
}

\author{
C.L. Edelstein ${ }^{1}$, M.K. Wickham ${ }^{2}$ and P.A. Kirby ${ }^{3}$ \\ ${ }^{1}$ Renal Unit, Department of Internal Medicine, and Departments of ${ }^{2}$ Anatomical Pathology and \\ ${ }^{3}$ Neuropathology, University of Stellenbosch and the Tygerberg Hospital, Private Bag, Tygerberg, 7505 , \\ Cape Province, South Africa
}

\begin{abstract}
Summary: A renal transplant patient who presented with a painful, proximal myopathy due to systemic calciphylaxis is described. The myopathy preceded the characteristic skin and soft tissue necrosis. Systemic calciphylaxis should be considered in a dialysis or a renal transplant patient presenting with a painful proximal myopathy even in the absence of necrotic skin lesions.
\end{abstract}

\section{Introduction}

Systemic calciphylaxis is a rare syndrome characterized by rapidly progressive ischaemic necrosis of large areas of skin and soft tissue associated with extensive vascular calcification..$^{1-3}$ The skin lesions are characterized by livedo reticularis or by dark red, tender, mottled areas on the thighs and buttocks that rapidly increase in size and ulcerate. It is described in chronic renal failure patients on dialysis and in renal transplant patients with or without hyperparathyroidism. ${ }^{4}$ It may respond to parathyroidectomy or withdrawal of immunosuppressive therapy..$^{5-7}$

We describe a case that is unusual in that the painful proximal myopathy preceded the characteristic skin lesions.

\section{Case report}

A 50 year old renal transplant patient presented in 1988 with painful proximal muscle weakness. In 1976 he developed chronic renal failure due to analgesic nephropathy and was started on haemodialysis. Four years later, he had an unsuccessful cadaver renal transplant. At this stage the patient already demonstrated features of metastatic calcification as evidenced by calcinosis cutis confirmed on skin biopsy. In 1982 a parathyroidectomy was done for secondary hyperparathyroidism. In addition to hyperplasia of the parathyroid glands, the small arteries surrounding the thyroid gland

Correspondence: C.L. Edelstein, M.B., Ch.B., M.Med., Renal Unit, Ward A7, Tygerberg Hospital, Private Bag, Tygerberg 7505, South Africa.

Accepted: 8 July 1991 demonstrated medial calcification and intimal proliferation (Figure 1). Five years after the parathyroidectomy he had a successful second cadaver renal transplant. In May 1988 he had pain and tenderness of the upper thigh muscles, but no muscle weakness was found. In November 1988 there was definite proximal muscle weakness. At this stage there were no necrotic skin lesions. He was on methylprednisolone $8 \mathrm{mg} /$ day and cyclosporin $150 \mathrm{mg}$ twice a day. Six months later he was confined to a wheelchair due to the muscle weakness.

Examination at this stage revealed two bullae on the skin of the lower leg surrounded by bruising and a few purpuric skin lesions on the upper leg. On neurological examination there was wasting and tenderness of the proximal arm and leg muscles.

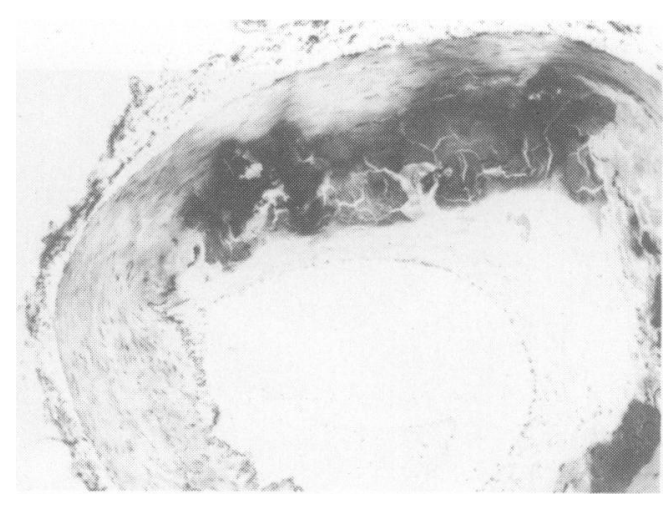

Figure 1 Small artery removed at parathyroidectomy (6 years before systemic calciphylaxis) demonstrating medical calcification, intimal thickening and fragmentation of the internal elastic lamina. (Vefhoef van Gieson $\times 100$ ). 
There was grade $4 / 5$ weakness of the proximal arm muscles and grade $2 / 5$ weakness of the proximal leg muscles. Tendon reflexes in the arms and knees were normal. Ankle reflexes were absent and there were signs of a sensorimotor peripheral neuropathy. Within 3 months the skin lesions on the lower leg had developed into large areas of skin necrosis (Figure 2).

The haemoglobin was $11.6 \mathrm{~g} / \mathrm{dl}$. The white blood cell count and differential count were normal. The serum creatinine was $150 \mu \mathrm{mol} / \mathrm{l}$ and the creatinine clearance was $42 \mathrm{ml} / \mathrm{min}$. The serum calcium was $2.35 \mathrm{mmol} / 1$ and the serum phosphate was 1.36 $\mathrm{mmol} / \mathrm{l}$. The parathyroid hormone level was $6 \mathrm{pmol} / 1$ (normal 1-5.5) (immunoradiometric assay of intact molecule). The creatine phosphokinase (MM fraction) was $600 \mathrm{U} / 1$ (normal 18-130). Thyroid functions were normal. X-rays of the hands and upper legs showed diffuse small vessel calcification.

Biopsy of the left vastus medialis muscle showed generalized atrophy of both Type 1 and 2 myofibres in keeping with an ischaemic myopathy (Figure 3 ) due to arterial calcification (Figure 4).

The patient underwent a left below knee amputation and recovered well, but he died unexpectedly a week post-operatively.

Post mortem histological examination of the ulcerated skin lesions over the right thigh demonstrated prominent medial calcification of small arteries in the subcutaneous fat with necrosis of the overlying epidermis. The coronary arteries showed marked medial calcification and intimal thickening with resultant luminal narrowing. Characteristic intimal plaques of atherosclerosis were absent. The lungs demonstrated extensive metastatic calcification within the alveolar walls. There was haemosiderin deposition, in keeping with transfusional iron overload, in the liver, spleen, pancreas, adrenal and thyroid gland.

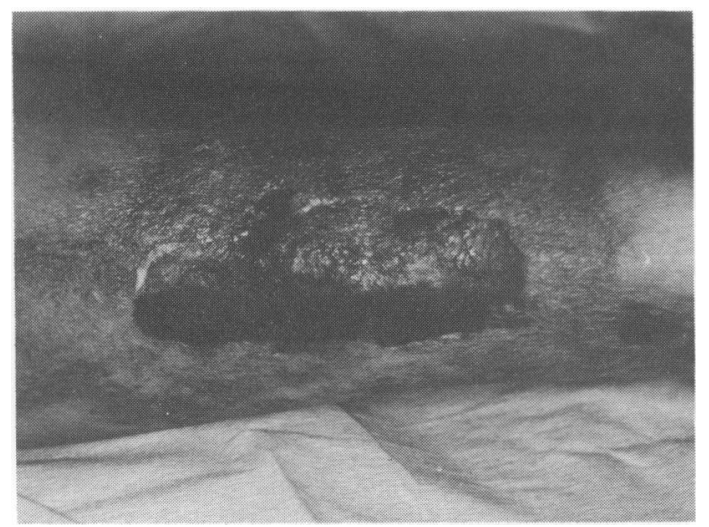

Figure 2 Necrotic skin lesions of the left lower leg.

\section{Discussion}

A painful proximal myopathy in systemic cal ciphylaxis due to muscle ischaemia and necrosis was first described by Richardson ${ }^{8}$ and later bల్ Goodhue. ${ }^{9}$ One patient had a fulminant myopath with myoglobinuria. Involvement of skeletal muse cle with muscle weakness, tenderness and/or sever鹿 myositis is now more frequently observed in systemic calciphylaxis in addition to the necrotient skin lesions. However the skin lesions usuallyo precede the myopathy.

All patients have widespread medial calcification and variable intimal proliferation of small to 
medium-sized arteries readily observed on biopsy of the affected part. This pathological feature is essential for the development of the syndrome. ${ }^{3}$ The diagnosis is made when there are the clinical features of ischaemia of skin and soft tissues as well as the vascular changes. It must be distinguished from other causes of vascular calcification, notably atherosclerosis which affects large and mediumsized arteries and morphologically demonstrates intimal fibrofatty plaques which may be complicated by ulceration and dystrophic calcification. Medial calcification may be a systemic manifestation of metastatic calcification secondary to any of a number of causes of disturbed calcium and/or phosphate metabolism but is distinct from calciphylaxis in that ischaemic necrosis is not a feature. It may also be seen as part of the normal ageing process as in Monckeberg's medial sclerosis which affects elderly patients with normal calcium metabolism. The vascular lesion is morphologically identical to that in calciphylaxis but again ischaemia is not a feature.

The pathogenesis of systemic calciphylaxis is obscure. Selye experimentally produced a similar condition in rats which he called calciphylaxis. ${ }^{10}$ Selye postulated that the tissues are first 'sensitized' by administration of parathyroid hormone, vitamin $D$, phosphate, calcium salts or by inducing renal failure. A 'critical period' elapses following which subsequent exposure of the rat to 'challenging agents' like local trauma, iron salts or egg albumin results in inflammation, necrosis and tissue calcification. Putative pathogenetic factors in the context of dialysis or renal transplant patients include disturbance of calcium or phosphate metabolism, uraemia, hypertension, transfusional iron overload, administration of vitamin D and immunosuppressive therapy. ${ }^{3}$ At least some of these factors were present in our case and the existence of medial calcification was documented some 6 years prior to the onset of skin necrosis. There may be an unknown factor, in someone with

\section{References}

1. Gipstein, R.M., Coburn, J.W., Adams, D.A. et al. Systemic calciphylaxis in man - a syndrome of tissue necrosis and vascular calcification in 11 patients with chronic renal failure. Arch Intern Med 1976, 136: 1273-1280.

2. Friedman, S.A., Novack, S. \& Thomson, G.E. Arterial calcification and gangrene in uremia. $N$ Engl J Med 1969, 280: $1392-1394$.

3. Wilkinson, S.P., Stewart, W.K., Parham, D.M. \& Guthrie, W. Symmetric gangrene of the extremities in late renal failure: A case report and review of the literature. $Q J \mathrm{Med} 1988,67$ : 319-341.

4. Asmundsson, P., Eliasson, G.J. \& Pordarson, H. A case of calciphylaxis. Scand J Urol Nephrol 1988, 22: 155-157.

5. Massry, S.G., Gordon, A., Coburn, J.W. et al. Vascular calcification and peripheral necrosis in a renal transplant recipient. Am J Med 1970, 49: 416-422. pre-existing vascular calcification, that precipitates the acute syndrome. ${ }^{6}$

The myopathy of systemic calciphylaxis is thought to be on an ischaemic basis via luminal narrowing produced by the medial calcification and intimal proliferation. However it is unlikely that simple mechanical vascular obstruction would improve following parathyroidectomy, without improvement of radiological vascular calcification, as is described. ${ }^{6}$

Prolonged administration of steroids may cause a myopathy. However we do not think it was the cause of the myopathy in our patient because of the relatively acute onset and rapid progression of the myopathy and histologically there was atrophy of both type 1 and type 2 myofibres and no myofibre lipid deposition. There were scanty degenerative and necrotic fibres suggesting on-going damage. In steroid-induced myopathies there is characteristic lipid deposition in type 1 myofibres and in longstanding steroid-induced myopathies there is mainly type 2 myofibre atrophy.

Calciphylaxis frequently runs a rapidly progressive course. Hyperparathyroidism and agents which enhance tissue catabolism like steroids and immunosuppressives in post-transplant patients can precipitate the syndrome. Vigorous normalization of the calcium phosphate product, parathyroidectomy, as well as withdrawal of immunosuppressive therapy in transplant patients can arrest or improve the lesions. ${ }^{6}$ Although therapy is not always successful, early recognition of this syndrome may permit effective therapy before the necrotic lesions became widespread.

Thus, in a patient receiving dialysis or a renal transplant patient presenting with a painful proximal myopathy, one should make a careful inspection for the characteristic skin lesions of systemic calciphylaxis. However, even in the absence of skin lesions and hyperparathyroidism, the diagnosis of calciphylaxis-induced myopathy should be entertained.
6. Adrogue, H.J., Frazier, M.R., Zeluff, B. \& Suki, W.N. Systemic calciphylaxis revisited. Am J Nephrol 1981, 1: 177-183.

7. Mehregan, D.A. \& Winkelman, R.K. Cutaneous gangrene, vascular calcification and hyperparathyroidism. Mayo Clin Proc 1989, 64: 211-215.

8. Richardson, J.A., Herron, G., Reitz, R. \& Layzer, R. Ischemic ulcerations of skin and necrosis of muscle in azotemic hyperparathyroidism. Ann Int Med 1969, 71: $129-138$.

9. Goodhue, W.W., Davis, J.N. \& Porro, R.S. Ischemic myopathy in uremic hyperparathyroidism. JAMA 1972, 221: 911-912.

10. Selye, H. Calciphylaxis. University of Chicago Press, Chicago, 1962. 